\title{
The role of voiding cystourethrography in the investigation of children with urinary tract infections
}

\author{
Linda C. Lee, MD;1 Armando J. Lorenzo, MD;,1,2 Martin A. Koyle, MD ${ }^{1,2}$ \\ The Hospital for Sick Children, Toronto, ON, Canada; ${ }^{2}$ Division of Urology, Department of Surgery, University of Toronto, Toronto, ON, Canada
}

Cite as: Can Urol Assoc J 2016;10(5-6):210-4. http://dx.doi.org/10.5489/cuai.3610

\section{Abstract}

Urinary tract infections (UTIs) represent a common bacterial cause of febrile illness in children. Of children presenting with a febrile UTI, 25-40\% are found to have vesicoureteral reflux (VUR). Historically, the concern regarding VUR was that it could lead to recurrent pyelonephritis, renal scarring, hypertension, and chronic kidney disease. As a result, many children underwent invasive surgical procedures to correct VUR. We now know that many cases of VUR are low-grade and have a high rate of spontaneous resolution. The roles of surveillance, antibiotic prophylaxis, endoscopic injection, and ureteral reimplantation surgery also continue to evolve. In turn, these factors have influenced the investigation of febrile UTIs.

Voiding cystourethrography (VCUG) is the radiographic test of choice to diagnose VUR. Due to its invasive nature and questionable benefit in many cases, the American Academy of Pediatrics (AAP) no longer recommends VCUG routinely after an initial febrile UTI. Nevertheless, these guidelines pre-date the landmark Randomized Intervention of Children with Vesicoureteral Reflux (RIVUR) trial and there continues to be controversy regarding the diagnosis and management of VUR. This paper discusses the current literature regarding radiographic testing in children with febrile UTIs and presents a practical risk-based approach for deciding when to obtain a VCUG.

\section{Introduction}

Urinary tract infections (UTIs) affect $2 \%$ of boys and $8 \%$ of girls by age seven. ${ }^{1}$ In febrile infants with no other identifiable cause, the prevalence of a UTI is approximately $7 \% .^{2}$ Of children presenting with febrile UTIs, 25-40\% are found to have vesicoureteral reflux (VUR). ${ }^{3,4}$ Historically, the default concern was that children with any degree of VUR had a substantially increased risk for recurrent pyelonephritis and secondary renal damage with sequelae of scarring, hypertension, and end-stage renal disease. ${ }^{5}$ This led to investigation and treatment for all.
Firstly, we now know that the majority of VUR cases are low-grade (i.e., Grades I-III) and have a high rate of spontaneous resolution. ${ }^{6}$ Secondly, a select group of low-risk patients could be managed with surveillance alone. ${ }^{7}$ Thirdly, while VUR is a risk factor for pyelonephritis and renal scarring, this association is not always present. In other words, some children present with pyelonephritis and subsequent renal scarring (or dysplasia) even without VUR. ${ }^{8}$ In these patients, the value of a voiding cystourethrography (VCUG) is sometimes questionable.

Management for VUR continues to evolve and is trending towards less aggressive options. ${ }^{9,10}$ The Randomized Intervention for Vesicoureteral Reflux (RIVUR) trial was a multicentre, placebo-controlled, double-blinded, randomized, control trial comparing placebo and antibiotic prophylaxis in children with Grades I-IV VUR. While antibiotic prophylaxis reduced UTI recurrence, it came at the cost of increased antibiotic resistance and had no statistically significant effect on renal scarring. ${ }^{11}$ The Swedish Reflux Trial compared antibiotic prophylaxis with endoscopic injection. In this trial, girls benefitted from both antibiotic prophylaxis and endoscopic injection. However, there was no difference among the two treatment groups in boys. ${ }^{12,13}$ Although it is not the only important outcome to consider, chronic kidney disease (CKD) was often used as justification for aggressive surgical intervention. However, the overall risk of a child with a UTI developing end-stage renal disease is less common than previously thought (an estimated 1 in 10 000). ${ }^{14}$ There is also a paucity of data to show that more aggressive surgical management will prevent CKD. In an Australian study, there was no decrease in reflux nephropathy over a 27 -year period following the introduction of reflux surgery. ${ }^{15}$ With increasing knowledge regarding the natural history of VUR and UTI, controversies have arisen regarding the diagnosis and management of these conditions in children.

Voiding cystourethrogram (VCUG), the gold standard radiographic test used to diagnose VUR, is a widely accepted test. However, it may also cause anxiety for patients and families due to its invasive nature, ${ }^{16}$ and carries a risk of radiation exposure ${ }^{17}$ and iatrogenic UTI. ${ }^{18}$ When weighing the 
risks of VCUG with the benefits of diagnosing a potentially benign condition, ordering this test has become individualized rather than a routine study. Recent guidelines regarding VCUG use have provoked debate and confusion for ordering physicians when compared to the past dogmatic approach.

\section{Controversies regarding the 2011 AAP guidelines}

In 2011, the American Academy of Pediatrics (AAP) published a revised guideline on the diagnosis and management of a febrile illness in children between ages of two and 24 months. ${ }^{19}$ It recommends that children presenting with an initial febrile UTI should have a renal and bladder ultrasound (RBUS). Only if there are abnormalities on RBUS (e.g., hydronephrosis, findings suggestive of scarring or suggestive of high-grade VUR) or after a second febrile UTI should patients be considered for a VCUG. It represents a dramatic departure from its recommendations in 1999, ${ }^{20}$ which advocated RBUS and VCUG after the initial febrile UTI. One major reason that it cites for limiting VCUG use is that its meta-analysis of randomized, controlled trials comparing antibiotic prophylaxis and placebo did not show a benefit with antibiotic prophylaxis. ${ }^{21-25}$ It also emphasized that its 2011 publication was before the RIVUR trial. ${ }^{11}$

The RIVUR trial found that antibiotic prophylaxis reduced recurrent febrile or symptomatic UTIs by $50 \%$ and the benefit was greater in those with a febrile index infection and bowel-bladder dysfunction. As mentioned earlier, the study found that antibiotic prophylaxis did not prevent renal scarring, although it was possibly underpowered to demonstrate this. The AAP's recommendations for limiting VCUG use hinged on the argument that antibiotic prophylaxis was not effective. The RIVUR authors directly challenged this and stated the following:

"As long as evidence supporting the benefit of prophylaxis was dubious, the recommendation of a watchfulwaiting approach, without performance of a voiding cystourethrographic study, seemed reasonable, because the imaging findings would not affect the nature of treatment. However, our finding that antimicrobial prophylaxis was associated with a reduced risk of recurrence may warrant reconsideration of that recommendation." 11

Another common critique of the AAP guidelines is that RBUS has supplanted VCUG as an initial "screening test." Although the authors do not explicitly refer to RBUS as a screening test, it is used in a similar manner. ${ }^{26}$ When a child presents with an initial febrile UTI, the AAP recommends obtaining a RBUS first. If the RBUS shows an abnormality or if the child presents with a second febrile UTI, then a VCUG is recommended. However, one may argue that RBUS is a poor screening test for VUR. Juliano et al performed a retrospective review of patients with a history of a febrile UTI, RBUS, and VCUG. ${ }^{27}$ They found that a quarter of patients with a normal RBUS had dilating (i.e., Grade III and above) VUR and $15 \%$ of those with a normal RBUS had recurrent pyelonephritis. Similarly, Nelson et al found that an abnormal RBUS had a low positive predictive value for VUR. ${ }^{26}$ The authors stated that VCUG and US should be viewed as complementary and that US does not replace VCUG as a screening test.

While cortical defects on dimercaptosuccinic acid (DMSA) scans suggest pyelonephritis in the acute phase and renal scarring or dysplasia in the chronic phase, DMSA provides information that RBUS and VCUG do not. The AAP guidelines notably exclude the role of nuclear scanning in the routine investigation of febrile UTIs. ${ }^{19}$ The authors assert that a DMSA scan in the acute setting rarely changes management and cites the radiation risk of a DMSA $(\sim 1 \mathrm{mSv})$, especially when adding it to a VCUG $(\sim 0.5-3.2 \mathrm{mSv}){ }^{28}$ A recent shortage of DMSA has affected hospitals worldwide, although the manufacturer expects the shortage to be resolved by August $2016 .{ }^{29}$ The AAP does not clearly advocate for either a classic top-down or bottom-up approach, ${ }^{30}$ as RBUS is essentially used as a screening test after the first febrile UTI. The debate regarding a top-down vs. bottom-up approach is beyond the scope of this paper.

The AAP guidelines refer to a "normal" RBUS, yet this term can be ambiguous. The authors suggest that a VCUG is indicated if a RBUS shows hydronephrosis, findings suggestive of renal scarring or suspicious of high-grade VUR. What is reported as urothelial thickening may be interpreted as abnormal, prompting a VCUG, or simply in keeping with an active infection. Although VCUG is the gold standard for diagnosing reflux, there is variability in protocols, reporting, and discordance amongst pediatric radiologists. ${ }^{31-33}$ Alexander and colleagues describe their protocol as, "two filling and voiding cycles in a non-sedated child. The contrast material is room temperature and infused from a height of approximately $70 \mathrm{~cm}$ above the pubic symphysis." ${ }^{34}$ Few studies involving VCUG explicitly report their protocol. Clinicians should be aware of the inconsistencies in the literature regarding VCUGs. On a final note, the preferences and values of the families are key factor in decisionmaking. As a guideline, it is not intended to be followed in every scenario and permits clinicians make decisions on a case-by-case basis.

\section{A risk-based approach to VCUG ordering}

When counselling families regarding VCUG use, one must consider the potential risks and benefits of ordering this test. On one hand, a VCUG could give the family more information about the cause of the febrile UTI(s), information about the degree and bilaterality of the VUR for risk stratification, expedite subsequent management (e.g.,watchful waiting, antibiotic prophylaxis, endoscopic injection, or 
Lee et al.

\section{Case study}

A one-year-old previously healthy girl presents with her first febrile UTI, diagnosed by a catheterized sample. She was seen in the local emergency department, given a treatment dose of antibiotics for a UTI. The urine culture later grew $E$. coli and she was started on prophylactic antibiotics (TMP/SMX) until her consultation in the urology clinic. A RBUS is normal.

\section{Do you order a VCUG?}

Proponents of performing a VCUG may cite the Swedish Reflux Trial, where antibiotic prophylaxis was shown to reduce the risk of UTI recurrence and renal scarring in girls with VUR, but not boys. ${ }^{12,13} \mathrm{~A}$ normal RBUS also does not exclude VUR. This child may go on to have another episode of pyelonephritis before a VCUG is ordered, thus delaying subsequent diagnosis and treatment. During the next episode, she may end up in the emergency department and possibly require hospitalization.

According to the 2011 AAP guidelines, this child would not get a VCUG at this point. ${ }^{19}$ While the AAP recommends prompt evaluation of a febrile illness, there is conflicting evidence as to whether this has an impact on renal scarring. ${ }^{35-37}$ Additionally, if a VCUG is ordered with the intention of continuing antibiotic prophylaxis in the setting of VUR, it is not supported by the RIVUR trial that prophylaxis prevents renal scarring. Ultimately, many patients in her situation would have a normal VCUG and this may not be worth the risk of radiation exposure (especially in girls, where the ovaries are in the radiation field), as well as the risk of iatrogenic UTI, distress, and invasiveness of urethral catheterization. With a VCUG after the initial febrile UTI, one may go down the road of diagnosing VUR and continues this child on antibiotic prophylaxis only to develop antibiotic resistance. In the RIVUR trial, the risk of recurrent febrile or symptomatic UTI was still only $23 \%$ on placebo. ${ }^{11}$

open ureteral reimplantation) and in doing so, potentially minimize the harm of a recurrent pyelonephritis. However, this represents the minority. Most patients will have either a negative VCUG or have low-grade, unilateral VUR with a high rate of spontaneous resolution and low risk of renal scarring. Rather than strict adherence to guidelines that predate the RIVUR trial, we recommend a risk-based approach to VCUG ordering and considering each patient on an individual basis. The following questions can be used to guide this complex decision-making process:

\section{What is the probability of VUR?}

The rate of VUR is estimated to be around $25-40 \%$ in patients presenting with a UTI. ${ }^{3,4}$ A more relevant question should be, "What is the probability of clinically significant VUR with a low chance of spontaneous resolution?" Factors affecting spontaneous resolution include age, gender, initial grade of VUR, renal scarring, bowel-bladder dysfunction, and bladder volume at onset of VUR. ${ }^{10,38}$ The VUR index is a simple tool to predict the chance of spontaneous resolution in children under two years of age and assigns a score depending on the gender, bladder volume at onset of VUR, ureteral anatomy, and initial VUR grade. ${ }^{39}$ In addition, other concomitant diagnoses may need to be considered and ruled out (e.g., posterior urethral valves, neurogenic bladder, etc.).

\section{What is the probability of major morbidity from VUR?}

Children who are sicker at initial infection, more medically fragile, or immunosuppressed potentially have a chance of incurring significant morbidity with recurrent pyelonephritis. Infants may be considered more vulnerable than older children, as signs and symptoms of pyelonephritis are often non-specific and infants may have more morbidity associated with a UTI. ${ }^{7}$ Conversely, some evidence suggests that the rate of renal scarring following pyelonephritis may be similar in infants and young children, compared to older children. ${ }^{37}$ In general, it is important to assess how reliable parents are, as it allows for prompt evaluation and management of a febrile UTI. Although the AAP guidelines recommend prompt evaluation of fever in children with a history of UTI, it is controversial whether early intervention actually impacts renal scarring. ${ }^{37}$

Prediction models can help to counsel parents on the risk of recurrent breakthrough UTIs based on factors such as high-grade VUR, presentation after a UTI, and female gender. ${ }^{40}$ Renal scarring can lead to hypertension and compromised renal function, especially in those with existing renal insufficiency. Again, we should consider that the chance of renal failure from UTI alone is lower than previously thought, an estimated 1 in 10000 patients presenting with a UTI. ${ }^{14}$ Overall, it is important to individualize therapies according to the patient and family factors.

\section{What if we find VUR on VCUG?}

If VUR is detected, some parents may have a strong desire to avoid antibiotic prophylaxis and/or surgical intervention and prefer watchful waiting until spontaneous resolution. Emerging awareness about antibiotic resistance is also a factor in this decision. ${ }^{11}$ As a result, it may not change management significantly to know about VUR unless the pyelonephritis is recurrent. In a case such as the one described here, the benefit of knowing about VUR and obtaining a VCUG is questionable.

\section{What if we don't find VUR on VCUG?}

As with any test, there is the possibility of over-investigation. In cases where the findings are normal, can one justify the risks of ordering a VCUG? On one hand, some families may benefit from the reassurance of a negative VCUG. On the other hand, VCUG does have a few notable risks. This includes potential distress and anxiety involving both patients and families. ${ }^{16,41}$ VCUG carries a risk of iatrogenic 
UTI both with (2\%) and without (6-22\%) antibiotic prophylaxis. ${ }^{18}$ Radiation exposure $(\sim 0.5-3.2 \mathrm{mSv})^{28}$ is also important to consider, as it potentially has long-term implications for infertility, cancer risk, etc. ${ }^{17}$ While VUR is a risk factor, it should be emphasized again that pyelonephritis can occur in the absence of VUR. ${ }^{8}$

\section{Conclusion}

As more is known about the natural history of VUR and alternatives to open surgery (i.e., surveillance, antibiotic prophylaxis, and endoscopic injection), VUR has moved from being a surgical condition to a medical one. The next era of VUR management will be about whom to screen for VUR. In many ways, VUR parallels the current debate surrounding prostate cancer facing our adult urology colleagues. It shares similar themes of over-diagnosis and over-treatment that affect many common conditions.

VUR represents a wide spectrum. The decision to order a VCUG should be based on weighing the potential risks and benefits. If a VCUG is recommended, there should be an expected benefit and not simply done to detect VUR. While guidelines help clinicians navigate through conflicting and confusing evidence, they are not applicable in every situation. It is important for clinicians to have knowledge of the current evidence and ask relevant questions, such as, "How does this change management?"

Competing interests: The authors declare no competing personal or financial interests.

\section{References}

1. Hellström A, Hanson E, Hansson S, et al. Association between urinary symptoms at 7 years old and previous urinary tract infection. Arch Dis Child 1991;66(2):232-4. http://dx.doi.org/10.1136/adc.66.2.232

2. Shaikh N, Morone NE, Lopez J, et al. Does this child have a urinary tract infection? JAMA 2007;298:2895904. http://dx.doi.org/10.1001/jama.298.24.2895

3. Greenfield SP, Wan J. Vesicoureteral reflux: Practical aspects of evaluation and management. Pediatr Nephrol 1996;10:789-94. hittp://dx.doi.org/10.1007/s004670050218

4. Hellerstein S. Urinary tract infections. Old and new concepts. Pediatr Clin North Am 1995;42:1433-57.

5. Arlen AM, Cooper CS. Controversies in the management of vesicoureteral reflux. Curr Urol Rep 16:64. http://dx.doi.org/10.1007/s11934-015-0538-2

6. Estrada CR, Passerotti CC, Graham DA, et al. Nomograms for predicting annual resolution rate of primary vesicoureteral reflux: Results from 2462 children. J Urol 2009;182:1535-41. http://dx.doi. org/10.1016/i.juro.2009.06.053

7. Peters CA, Skoog SJ, Arant BS Jr, et al. Summary of the AUA guideline on management of primary vesicoureteral reflux in children. J Urol 2010;184:1134-44. http://dx.doi.org/10.1016/i.juro.2010.05.065

8. Shaikh N, Ewing AL, Bhatnagar $S$, et al. Risk of renal scarring in children with a first urinary tract infection: A systematic review. Pediatrics 2010;126:1084-91. http://dx.doi.org/10.1542/peds.2010-0685

9. Herbst KW, Corbett ST, Lendvay TS, et al. Recent trends in the surgical management of primary vesicoureteral reflux in the era of dextranomer/hyaluronic acid. J Urol 2014;191:1628-33. http://dx.doi. org/10.1016/i.jur. 2013.09.055

10. Koyle MA, Elder JS, Skoog SJ, et al. Febrile urinary tract infection, vesicoureteral reflux, and renal scarring: Current controversies in approach to evaluation. Pediatr Surg Int 2011;27:337-46. http://dx.doi. org/10.1007/s00383-011-2863-y

11. The RIVUR Trial Investigators, Hoberman A, Greenfield SP, et al. Antimicrobial prophylaxis for children with vesicoureteral reflux. N Engl J Med 2014;370:2367-76. http://dx.doi.org/10.1056/NEJMoal 401811
12. Brandström P, Esbiörner E, Herthelius $M$, et al. The Swedish Reflux Trial in Children: III. Urinary tract infection pattern. J Urol 2010;184:286-91. http://dx.doi.org/10.1016/i.juro.2010.01.061

13. Brandström P, Esbjörner E, Herthelius $M$, et al. The Swedish Reflux Trial in Children: IV. Renal damage. J Urol 2010;184:292-7. http://dx.doi.org/10.1016/i.juro.2010.01.060

14. Craig JC, Williams GJ. Denominators Do Matter: It's a myth — urinary tract infection does not cause chronic kidney disease. Pediatrics 2011;128:984-5. http://dx.doi.org/10.1542/peds.2011-2631

15. Craig JC, Irwig LM, Knight JF, et al. Does treatment of vesicoureteric reflux in childhood prevent endstage renal disease attributable to reflux nephropathy? Pediatrics 2000;105:1236-41. http://dx.doi. org/10.1542/peds.105.6.1236

16. Lachenmyer LL, Anderson JJ, Clayton DB, et al. Analysis of an intervention to reduce parental anxiety prior to voiding cystourethrogram. J Pediatr Urol 2013;9:1223-8. http://dx.doi.org/10.1016/i. jpurol.2013.05.015

17. Perisinakis K, Raissaki M, Damilakis J, et al. Fluoroscopy-controlled voiding cystourethrography in infants and children: Are the radiation risks trivial? Eur Radiol 2006;16:846-51. http://dx.doi.org/10.1007/ s00330-005-0072-6

18. Rachmiel $M$, Aladjem $M$, Starinsky R, et al. Symptomatic urinary tract infections following voiding cystourethrography. Pediatr Nephrol 2005;20:1449-52. http://dx.doi.org/10.1007/s00467-005-1942-5

19. Roberts KB and Subcommittee on Urinary Tract Infection, Steering Committee on Quality Improvement and Management. Urinary tract infection: Clinical practice guideline for the diagnosis and management of the initial UTl in febrile infants and children 2 to 24 months. Pediatrics 2011;128:595-610. http:// dx.doi.org/10.1542/peds.2011-1330

20. Committee on Quality Improvement, Subcommittee on Urinary Tract Infection. Practice parameter: The diagnosis, treatment, and evaluation of the initial urinary tract infection in febrile infants and young children. Pediatrics 1999; 103:843-52.

21. Garin EH, Olavarria F, Garcia Nieto V, et al. Clinical significance of primary vesicoureteral reflux and urinary antibiotic prophylaxis after acute pyelonephritis: A multicentre, randomized, controlled study. Pediatrics 2006;117:626-32. http://dx.doi.org/10.1542/peds.2005-1362

22. Montini $G$, Rigon L, Zucchetta $P$, et al. Prophylaxis after first febrile urinary tract infection in children? A multicentre, randomized, controlled, non-inferiority trial. Pediatrics 2008;122:1064-71. http://dx.doi. org/10.1542/peds.2007-3770

23. Craig JC, Simpson JM, Williams $G$ J, et al. Antibiotic prophylaxis and recurrent urinary tract infection in children. N Engl J Med 2009;36:1748-59. http://dx.doi.org/10.1056/NEJMoa0902295

24. Roussey-Kesler G, Gadjos V, Idres N, et al. Antibiotic prophylaxis for the prevention of recurrent urinary tract infection in children with low-grade vesicoureteral reflux: Results from a prospective, randomized study. J Urol 2008;179:674-9. http://dx.doi.org/10.1016/i.juro.2007.09.090

25. Pennesi $M$, Travan $L$, Peratoner $L$, et al. Is antibiotic prophylaxis in children with vesicoureteral reflux effective in preventing pyelonephritis and renal scars? A randomized, controlled trial. Pediatrics 2008;121:e1489-94. http://dx.doi.org/10.1542/peds.2007-2652

26. Nelson $\mathrm{CP}$, Johnson EK, Logvinenko $\mathrm{T}$, et al. Ultrasound as a screening test for genitourinary anomalies in children with UTI. Pediatrics 2014;133:e394-403. http://dx.doi.org/10.1542/peds.2013-2109

27. Juliano TM, Stephany HA, Clayton DB, et al. Incidence of abnormal imaging and recurrent pyelonephritis after first febrile urinary tract infection in children 2 to 24 months old. J Urol 2013;190:1505-10. hittp:// dx.doi.org/10.1016/i.juro.2013.01.049

28. La Scola C, De Mutiis C, Hewitt IK, et al. Different guidelines for imaging after first UTI in febrile infants: Yield, cost, and radiation. Pediatrics 2013;131:e665-71. http://dx.doi.org/10.1542/peds.2012-0164

29. FDA drug shortages: Current and resolved drug shortages and discontinuations reported to FDA. Silver Spring: U. S. Department of Health \& Human Services: c2014-2015 [updated 2015 Jul 15; cited 2016 Jan 26]. httrp://www.accessdata.fda.gov/scripts/drugshortages/dsp_ActivelngredientDetails.fm?Al=Technetium\%20 Tc99m\%20Succimer\%20Iniection\%20(DMSA) \&st=c\&tab=tabs-1. Accessed January 26, 2016.

30. Prasad MM, Cheng EY. Radiographic evaluation of children with febrile urinary tract infection: Bottom-up, top-down, or none of the above? Adv Urol 2012;2012:716739.

31. Palmer BW, Ramii FG, Snyder CT, et al. Voiding cystourethrogram — are our protocols the same? J Urol 2011;186:1668-71. http://dx.doi.org/10.1016/i.juro.2011.04.006

32. Schueffer AJ, Sood S, Logvinenko $T$, et al. Variation in the documentation of findings in pediatric voiding cystourethrogram. Pediatr Radiol 2014;44:1548-56. http://dx.doi.org/10.1007/s00247-014-3028-7

33. Greenfield SP, Carpenter MA, Chesney RW, et al. The RIVUR voiding cystourethrogram pilot study: Experience with radiologic reading concordance. J Urol 2012;188:1608-12. http://dx.doi. org/10.1016/i.juro.2012.06.032

34. Alexander SE, Arlen AM, Storm DW, et al. Bladder volume at onset of vesicoureteral reflux is an independent risk factor for breakthrough febrile urinary tract infection. J Urol 2015;193:1342-6. http://dx.doi. org/10.1016/i.juro.2014.10.002

35. Winter AL, Hardy BE, Alton DJ, et al. Acquired renal scars in children. J Urol 1983;129:1190-4.

36. Smellie JM, Poulton A, Prescod NP. Retrospective study of children with renal scarring associated with reflux and urinary infection. BMJ 1994;308:1193-6. http://dx.doi.org/10.1136/bmi.308.6938.1193 
37. Hewitt IK, Zucchetta $P$, Rigon $L$, et al. Early treatment of acute pyelonephritis in children fails to reduce scarring: Data from the Italian Renal Infection Study Trials. Pediatrics 2008;122:486-90. http://dx.doi. org/10.1542/peds.2007-2894

38. Knudson MJ, Austin JC, MCMillan ZM, et al. Predictive factors of early spontaneous resolution in children with primary vesicoureteral reflux. J Urol 2007;178:1684-8. http://dx.doi.org/10.1016/i. juro.2007.03.161

39. Arlen $\mathrm{AM}$, Garcia-Roig $M$, Weiss $A D$, et al. Vesicoureteral reflux index: 2-institution analysis and validation. J Urol 2016;195:1-6. http://dx.doi.org/10.1016/i.juro.2015.03.094

40. Hidas $\mathrm{G}$, Billimek J, Nam A, et al. Predicting the risk of breakthrough urinary tract infections: Primary vesicoureteral reflux. J Urol 2015;194:1396-401. http://dx.doi.org/10.1016/i.juro.2015.06.019

41. Srivastava $T$, Betts $G$, Rosenberg AR, et al. Perception of fear, distress, and pain by parents of children undergoing a micturating cystourethrogram: A prospective study. J Paediatr Child Health 2001;37:271-3. http://dx.doi.org/10.1046/i.1440-1754.2001.00663.x

Correspondence: Dr. Martin Koyle, Pediatric Urology, The Hospital for Sick Children, Toronto, ON, Canada; Martin.koyle@sickkids.ca

\section{${ }^{\circ} \mathrm{XGEVA}$ \\ (denosumab)}

Indication and clinical use:

- XGEVA ${ }^{\circledR}$ is indicated for reducing the risk of developing skeletal-related events (SREs) in patients with bone metastases from breast cancer, prostate cancer, non-small cell lung cancer, and other solid tumours.

- Not indicated for reducing the risk of developing skeletal-related events in patients with multiple myeloma.

- Not indicated for reducing the risk of developing skeletal-related events in pediatric patients.

\section{Contraindications:}

- In patients with pre-existing hypocalcemia, which must be corrected prior to initiation.

\section{Most serious warnings and precautions:}

Osteonecrosis of the jaw (ONJ): In clinical trials, the incidence of ONJ was higher with longer duration of exposure. In patients with risk factors for $0 \mathrm{NJ}$, an individual risk/benefit assessment should be performed before initiating therapy with XGEVA. An oral exam should be performed and a dental exam with appropriate preventive dentistry is recommended prior to treatment with XGEVA, especially in patients with risk factors for ONJ. Avoid invasive dental procedures while receiving XGEVA. In patients who develop ONJ during treatment with XGEVA, a temporary interruption of treatment should be considered based on individual risk/benefit assessment until the condition resolves.

\section{Other relevant warnings and precautions:}

-Do not use concurrently with Prolia

- Do not use concurrently with bisphosphonates

- Hypocalcemia has been reported (including severe symptomatic hypocalcemia and fatal cases). Monitor calcium prior to the initial dose, within two weeks after the initial dose, and if suspected symptoms of hypocalcemia occur. Administer adequate calcium, vitamin $\mathrm{D}$, and magnesium, as necessary. If hypocalcemia occurs while receiving XGEVA, additional short-term calcium supplementation and additional monitoring may be necessary.

- Caution on risk of hypocalcemia and accompanying increases in parathyroid hormone in patients with renal impairment

- Skin infections

- Hypersensitivity reactions including anaphylaxis

- Atypical femoral fractures

- Not recommended for use in pregnant women. Women should not become pregnant during treatment and for at least 5 months after the last dose of XGEVA.

\section{For more information:}

Please consult the Product Monograph at

http://www.amgen.ca/Xgeva_PM.pdf for important information relating to adverse reactions, drug interactions, and dosing that have not been discussed here.

The Product Monograph is also available by calling Amgen Medical Information at 1-866-502-6436.

Fizazi et al. study ${ }^{2}$

double-blind, double-dummy, active-controlled study Patients with castrate-resistant prostate cancer and bone metastases ( $\mathrm{n}=1901)$ received either $120 \mathrm{mg}$ XGEVA SC Q4W (once every 4 weeks) $(n=950)$ or $4 \mathrm{mg}$ zoledronic acid IV Q4W ( $n=951)$. The primary outcome measure was to demonstrate non-inferiority of time to first on-study SRE as compared to zoledronic acid. The secondary outcome measures were superiority of time to first on-study SRE and superiority of time to first and subsequent SREs. An SRE is defined as any of the following: pathologic fracture radiation therapy to bone, surgery to bone or spinal cord compression.

References:

1. XGEVA $\oplus$ Product Monograph, Amgen Canada, 2015

2. Fizazi $\mathrm{K}$, et al. Denosumab versus zoledronic acid for treatment of bone metastases in men with castration-resistant prostate cancer: a randomized, double-blind study. Lancet. 2011;377(9768):813-822.
AMCEN

Oncology
(C) 2016 Amgen Canada Inc. All rights reserved.

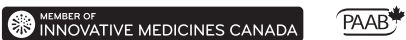

